\section{Oxygen availability and tumors: a tricky relationship}

The findings of Galluzzo et al. (4) not only provide additional insights into the role of the tumor microenvironment and oxygen in tumorigenesis, but they also raise additional questions regarding our understanding of oxygen metabolism and tumorigenesis. For example, it has been documented that high-altitude populations, subject to chronic hypoxia, tend to have a lower frequency of cancer (17). Is this because there is an adaptation that increases oxygen transport into tissues (e.g., enhanced $\mathrm{Mb}$ expression; ref. 18) or because oxygen deprivation is antitumorigenic? In addition, it is known that animals with larger body mass tend to have a lower incidence of cancer development (19), and yet the tissues from these animals have lower specific metabolic rates and oxygen consumption (20). Notwithstanding these intriguing nuances, the work of Galluzzo and coworkers clearly provides additional food for our collective thought on tumor metabolism and tumorigenesis.

Address correspondence to: Ulrich Flögel, Institut für Herz- und Kreislaufphysiologie, Heinrich-Heine-Universität,
Universitätsstrasse 1, 40225 Düsseldorf, Germany. Phone: 49-211-8112785; Fax: 49211-8112672; E-mail: floegel@uni-duesseldorf.de. Or to: Chi Dang, Johns Hopkins Medicine, Ross Building, Room 1032, 720 Rutland Avenue, Baltimore, Maryland 21205. Phone: (410) 955-2411; Fax: (410) 955-0185; E-mail: cvdang@jhmi.edu.

1. Hsu, P.P., and Sabatini, D.M. 2008. Cancer cell metabolism: Warburg and beyond. Cell. 134:703-707.

2. Jain, R.K. 2005. Normalization of tumor vasculature: an emerging concept in antiangiogenic therapy. Science. 307:58-62.

3. Dewhirst, M.W., Cao, Y., and Moeller, B. 2008. Cycling hypoxia and free radicals regulate angiogenesis and radiotherapy response. Nat. Rev. Cancer. 8:425-437.

4. Galluzzo, M., Pennacchietti, S., Rosano, S., Comoglio, P.M., and Michieli, P. 2009. Prevention of hypoxia by myoglobin expression in human tumor cells promotes differentiation and inhibits metastasis. J. Clin. Invest. 119:865-875.

5. Wittenberg, J.B., and Wittenberg, B.A. 2003. Myoglobin function reassessed. J. Exp. Biol. 206:2011-2020.

6. Ordway, G.A., and Garry, D.J. 2004. Myoglobin: an essential hemoprotein in striated muscle. J. Exp. Biol. 207:3441-3446.

7. Gödecke, A. 2006. On the impact of NO-globin interactions in the cardiovascular system. Cardiovasc. Res. 69:309-317.

8. Burmester, T., Gerlach, F., and Hankeln, T. 2007. Regulation and role of neuroglobin and cytoglobin under hypoxia. Adv. Exp. Med. Biol. 618:169-180.

9. Wink, D.A., Ridnour, L.A., Hussain, S.P., and Harris, C.C. 2008. The reemergence of nitric oxide and cancer. Nitric Oxide. 19:65-67.

10. Quintero, M., Brennan, P.A., Thomas, G.J., and Moncada, S. 2006. Nitric oxide is a factor in the stabilization of hypoxia-inducible factor-1alpha in cancer: role of free radical formation. Cancer Res. 66:770-774.

11. Simon, M.C. 2006. Mitochondrial reactive oxygen species are required for hypoxic HIF alpha stabilization. Adv. Exp. Med. Biol. 588:165-170.

12. Gloster, J., and Harris, P. 1977. Fatty acid binding to cytoplasmic proteins of myocardium and red and white skeletal muscle in the rat. A possible new role for myoglobin. Biochem. Biophys. Res. Commun. 74:506-513.

13. Götz, F.M., Hertel, M., and Groschel-Stewart, U. 1994. Fatty acid binding of myoglobin depends on its oxygenation. Biol. Chem. Hoppe Seyler. 375:387-392.

14. Gatenby, R.A., and Gillies, R.J. 2004. Why do cancers have high aerobic glycolysis? Nat. Rev. Cancer. 4:891-899.

15. Dang, C.V., Kim, J.W., Gao, P., and Yustein, J. 2008. The interplay between MYC and HIF in cancer. Nat. Rev. Cancer. 8:51-56.

16. Shivapurkar, N., et al. 2008. Cytoglobin, the newest member of the globin family, functions as a tumor suppressor gene. Cancer Res. 68:7448-7456.

17. Weinberg, C.R., Brown, K.G., and Hoel, D.G. 1987. Altitude, radiation, and mortality from cancer and heart disease. Radiat. Res. 112:381-390.

18. Terrados, N. 1992. Altitude training and muscular metabolism. Int. J. Sports Med. 13(Suppl. 1): S206-S209.

19. Nagy, J.D., Victor, E.M., and Cropper, J.H. 2007. Why don't all whales have cancer? A novel hypothesis resolving Peto's paradox. Integr. Comp. Biol. 47:317-328. doi:10.1093/icb/icm062.

20. Savage, V.M., et al. 2007. Scaling of number, size, and metabolic rate of cells with body size in mammals. Proc. Natl. Acad. Sci. U. S. A. 104:4718-4723.

\title{
Kallikreins and lupus nephritis
}

\section{Claudio Ponticelli' and Pier Luigi Meroni²}

${ }^{1}$ Division of Nephrology, IRCCS Istituto Clinico Humanitas, Milan, Italy. ${ }^{2}$ Clinical Immunology and Rheumatology Unit, Department of Internal Medicine, IRCCS Istituto Auxologico Italiano, University of Milan, Milan, Italy.

The kidney kallikrein-kinin system plays important roles in inflammation, coagulation, angiogenesis, and regulation of vessel tone and permeability. In this issue of the JCI, Liu et al. provide data that suggest a protective role for kallikrein in animal models of anti-glomerular basement membrane (GBM) antibody-induced nephritis, an experimental model of Goodpasture disease (see the related article beginning on page 911). Furthermore, human systemic lupus erythematosus and lupus nephritis were shown to be associated with kallikrein 1 (KLK1) and the $K L K 3$ promoter. The authors suggest that kallikrein genes are involved in the development of SLE and lupus nephritis and may exert a renoprotective role. It is possible, however, that the kallikrein-kinin system may play dual roles: protecting the kidney against ischemia and interstitial fibrosis while also mediating vasodilation, inflammation, and activation of the innate immune response.

Conflict of interest: C. Ponticelli is a consultant for Novartis, Italy.

Nonstandard abbreviations used: ACE, angiotensin converting enzyme; GBM, glomerular basement membrane; KLK1, kallikrein 1.

Citation for this article: J. Clin. Invest. 119:768-771 (2009). doi:10.1172/JCI38786.

\section{The kallikrein-kinin system}

The kallikrein-kinin system comprises proteins that play a role in inflammation, coagulation, and regulation of vessel tone and permeability via the production of small peptides called kinins. The system originates from prekallikrein, a serum protease that, after being activated by factor XIIa (also known as Hageman factor), is cleaved to form kallikrein. Kallikreins are serine peptidases (kininogenases) that rapidly release kinins in the plasma by cleaving kininogens - multifunctional proteins derived mainly from $\alpha_{2}$-globulins. Tissue kallikrein 1 (KLK1) is synthesized in many organs, including kidney and arteries, where it can generate the vasodilators bradykinin and kallidin, which are rapidly hydrolyzed to inactive products by a group of peptidases known as kininases (Figure 1).

Kallikreins are encoded by a variable number of genes in different mammalian species. The human tissue kallikreins are encoded by a cluster of 15 genes located on chromosome 19q13.4, a position analogous to that of the kallikrein gene family 


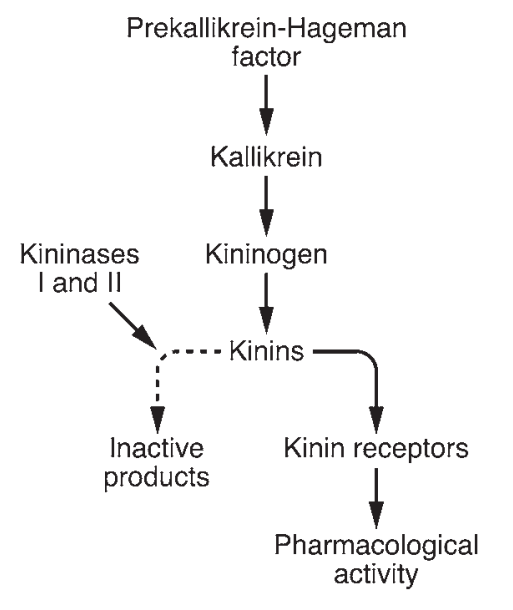

on mouse chromosome $7(1,2)$. Among these genes, the principal kinin-generating enzyme, KLK1, is encoded by KLK1.

Complement activation may trigger the kinin cascade and vice versa. There is crosstalk between the two systems, which leads to the generation of activation products that ultimately affect endothelial functions (3).

\section{Kallikreins, hypertension, and renal function}

Experimental and clinical studies have shown an inverse correlation between urinary kallikrein levels and blood pressure (4-6). Further studies have shown that the tissue kallikrein-kinin system can inhibit apoptosis, proliferation, hypertrophy, and fibrosis and can promote angiogenesis in different experimental animal models (7). Moreover, kallikrein gene delivery or kallikrein protein infusion improves cardiac, renal, and neurological function without reducing blood pressure (7). A main player involved in these effects is likely KLK1. Studies of KLK1-deficient mice and human subjects partially deficient in KLK1 have revealed a critical role for KLK1 in arterial function in both species and prompted a search for a possible genetic link between kallikrein gene polymorphisms and blood pressure regulation (8). In an effort to identify a genetic basis for differential urinary kallikrein excretion, Yu et al. (6) found that the KLK1 promoter is uniquely polymorphic, with a poly-G-length polymorphism coupled with multiple singlebase substitutions. These authors also found a significant association between the $12 \mathrm{G}$ allele (the longest of the length locus alleles) and arterial hypertension and end-stage renal disease in African Americans (6). These findings suggested that

\section{Figure 1}

Simplified overview of the kallikrein-kinin system. Kallikreins originate from prekallikrein, which is cleaved to form kallikrein after being activated by factor XIla (Hageman factor). Kallikreins are enzymes that cleave kininogens (proteins derived mainly from $\alpha_{2}$-globulins) into peptides called kinins. In turn, kinins may be cleaved by kininases to form inactive final products or may bind to their receptors and exert pharmacological activity.

kallikrein/kinin may serve as new drug targets for the prevention and treatment of the systemic vasculopathy associated with arterial hypertension.

\section{Kallikreins, SLE, and lupus nephritis}

Kallikreins can also act as proinflammatory mediators and play a role in several autoimmune diseases. SLE is a prototypic systemic autoimmune disease of unknown etiology in which immune complex deposition and complement activation lead to inflammation and tissue damage. The kidney is a target organ of such processes, and immune-mediated nephritis is a common complication of SLE. The SLE autoimmune response involves abnormal expansion of autoreactive T and B cells. An important role is also played by innate immune effectors, which trigger additional local mechanisms of inflammation and tissue damage (9-11) (Figure 2).

Theoretically, abnormalities in angiotensin-converting enzyme (ACE), a kininase that breaks down kallikrein-produced kinins, might favor perpetuation of autoimmune inflammation and progression of renal disease in SLE. Some studies have suggested an association between SLE and an insertion/deletion (I/D) polymorphism in ACE. Individuals homozygous for the insertion polymorphism display higher plasma levels of ACE and show significantly increased activity of lupus nephritis and SLE, suggesting a possible pathogenic role of kallikreins in SLE (12). However, other studies could not confirm such an association, and a metaanalysis of 2,962 patients showed a lack of association of the ACE I/D polymorphism with SLE and lupus nephritis (13).

In line with the role of kallikreins as proinflammatory mediators, higher plasma levels of high-MW kininogen, low-MW kininogen, and kallikrein were found in 30 patients with active lupus nephritis compared with age- and sex-matched controls (14). In addition, plasma and urinary activities of tissue kallikrein and kininase II were also significantly increased in these patients. These data suggest a role for the kallikrein-kinin system in the acute manifestations of lupus nephritis.

\section{$K L K 1$ and $K L K 3$ are disease genes in lupus and anti-GBM antibody- induced nephritis}

Anti-glomerular basement membrane (GBM) antibodies have been used to induce experimental nephritis in mice (15). It should be noted, however, that inbred mouse strains differ in their susceptibility to anti-GBM antibody-induced nephritis and lupus nephritis. In this issue of the JCI, Liu et al. set out to investigate whether kallikreins play a role in this differential susceptibility to renal disease. The authors report that inbred mouse strains with upregulated expression of renal and urinary kallikreins exhibited less susceptibility to anti-GBM antibody-induced and lupus nephritis (16). The authors also showed that the administration of kallikreins dampened anti-GBM antibody-induced nephritis, strongly suggesting a link between kallikreins and immune complex-mediated kidney damage. In line with their demonstration that Klk genes from one of the nephritissensitive mouse strains conferred increased nephritis susceptibility in disease-resistant mice, the authors found that SLE and lupus nephritis in human patients were also associated with genes homologous to murine Klk genes, particularly KLK1 and the KLK3 (also called KLKB1) promoter. Taken together, these results suggest that kallikrein genes $K L K 1$ and $K L K 3$ are disease genes in lupus and anti-GBM antibodyinduced nephritis (16).

\section{Kallikreins in lupus nephritis: a dual role?}

The elegant study reported by Liu et al. represents an important advance and may pave the way for new diagnostic and therapeutic approaches for lupus nephritis (16). One of the main findings of the study is that effector mechanisms of the innate immune response may play a role in modulating the autoimmune response against a target organ (i.e., the kidney) (16). In fact, the authors raise the possibility that the kallikrein-kinin system activated by the innate immune response enhances the local tissue damage triggered by the autoimmune response. 


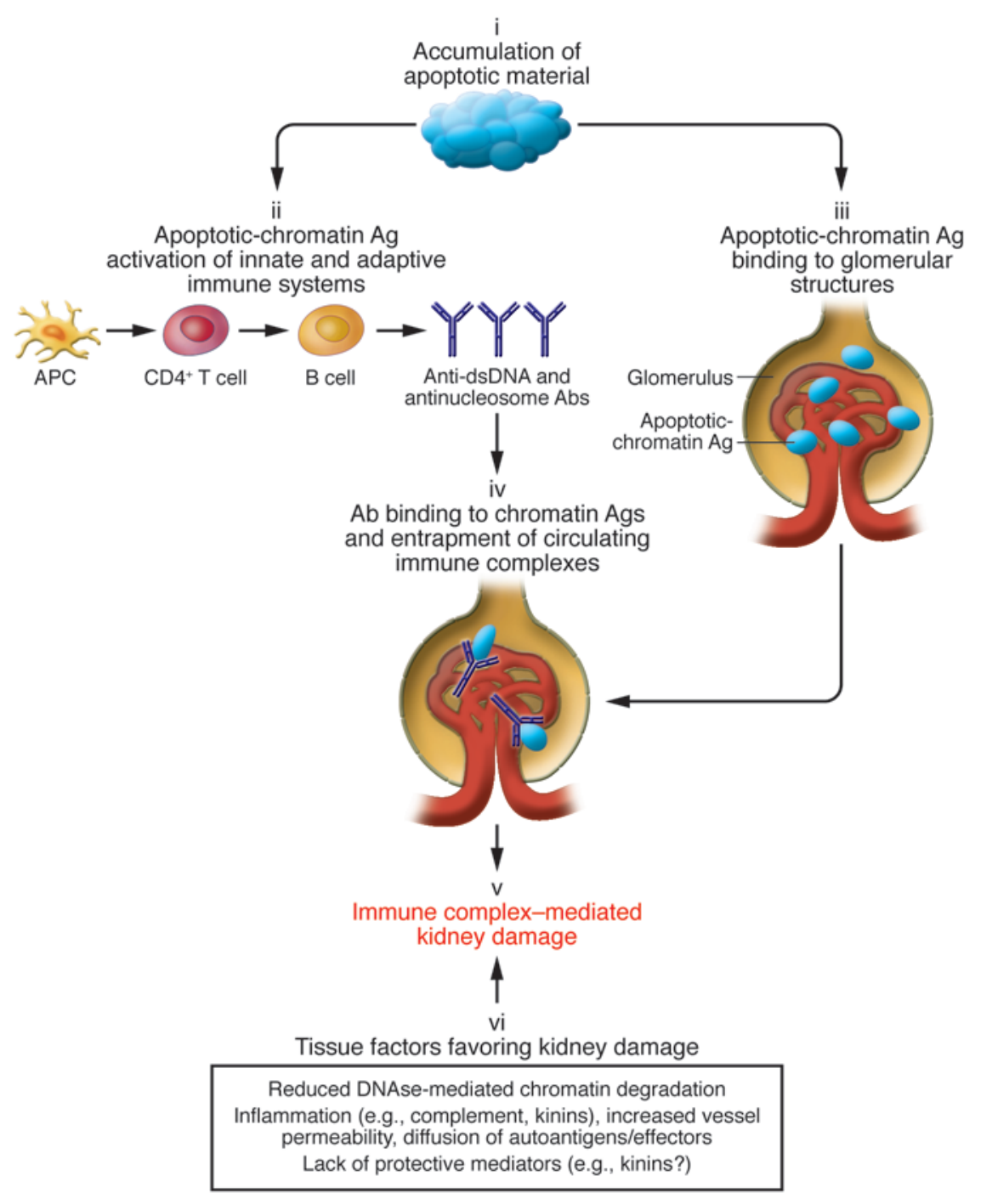

However, a number of issues remain unresolved. The experimental data are largely derived from evidence that a mouse model of anti-GBM antibody-induced glomerulonephritis may mimic lupus nephritis (16). Of the approximately 25 different molecules that have been specifically examined in the experimental anti-GBM antibody-induced disease model and also in spontaneous lupus nephritis, all were reported to influence both diseases concordantly, suggesting that the experimental model might be a useful tool for unraveling the molecular basis of spontaneous lupus nephritis (15). Although Liu et al. (16) also showed an association between kallikrein genes and human SLE and lupus nephritis, there are still discrepancies between the mouse model and human diseases: (a) the target of anti-GBM antibodies in human anti-GBM antibody-induced disease (also known as Goodpasture disease) is the $\alpha 3$ chain of type IV collagen, while at least nine antigens, of which the most frequent are dsDNA and nucleosomes, have been identified in SLE (17); (b) in Goodpasture disease, there are linear deposits of IgG and C3 along the GBM, while in human lupus nephritis, immunofluorescence shows subendothelial, mesangial, or subepithelial granular deposits of IgG, C3, and C1q, and more rarely of IgA and IgM (18); (c) the initial immunological response in each condition and also the downstream pathogenic cascades are different, as shown by the diverse clinical and histological patterns observed in the two diseases. In particular, in human lupus nephritis, six classes of glomerular lesions, each containing different subclasses, have been recognized (18). A variety of factors may be responsible for such a heterogeneous renal pathology. The

\section{Figure 2}

The main mechanisms mediating kidney damage in lupus nephritis. SLE is characterized by an accumulation of apoptotic material due to its poor clearance (9) (i). This leads to an immune response against chromatin (ii) and to increased expression and binding of apoptotic-chromatin antigens to kidney glomerular structures (iii). Antibodies against apoptoticchromatin antigens (in particular anti-dsDNA and anti-nucleosome antibodies) may form immune complexes in the kidney with their specific planted antigens or may be entrapped on glomerular structures as immune complexes preformed in the circulation (iv). Immune complex deposition/formation eventually leads to immune-mediated tissue inflammation and damage (v). Local factors may also increase the susceptibility of renal tissue to damage (vi): Chromatin antigens may accumulate in the kidney tissue because of reduced DNAsemediated chromatin degradation (11), and immune-mediated inflammation may itself increase vessel permeability, consequently increasing diffusion of the autoantigens themselves and of the soluble and cellular mediators of the autoimmune response. In this context, the lack of the potentially protective effect of kinins may represent an additional mechanism contributing to renal tissue damage. Ag, antigen. morphologic type of glomerulonephritis and its clinical course may depend upon the predominant type of antigen exposed, its persistence, the concurrent degree of activation of complement, the recruitment of polymorphonuclear cells and macrophages, the stimulation of the clotting system, as well as the development of hypertension and the functional response of the kidney to reduced renal mass. Further studies are necessary to assess the relative contribution to disease of polymorphisms in $K L K 1$ or the $K L K 3$ promoter; many other mediators, including C1-inhibitor - the physiological inhibitor of the plasma kallikrein and kinin signaling pathways - may also play a role.

On the other hand, one may wonder whether the involvement of the kallikreinkinin system is actually beneficial in active lupus nephritis. As discussed above, previ- 
ous studies have reported a nephroprotective effect of the tissue kallikrein-kinin system $(7,8)$. However, in the inflammatory response, the kallikrein proteolytic cascade plays a significant role, since it is considered to initiate and maintain systemic inflammatory responses and immune-modulated disorders (3). Moreover, kinins may also facilitate immune complex deposition by vasodilation and may induce the release of other proinflammatory mediators, including cytokines $(3,19)$. Finally, formation of bradykinin has been reported to activate innate immunity (20), which is actively involved in the pathogenesis of SLE (9). Thus, it seems that in lupus nephritis, the kallikrein-kinin system may operate as either a mediator or modulator of immunomediated inflammation. It might be possible that kallikreins actually protect the kidney in the quiescent phases of the disease, when arterial hypertension and chronic renal failure are responsible for progressive interstitial fibrosis and glomerular sclerosis, but they could be potentially harmful in the acute phases of the disease, when the immune and inflammatory responses take place. Alternatively, KLK downregulation during acute kidney inflammation may represent a form of negative feedback to counteract the tissue damage.

These uncertainties do not reduce the importance of the instructive contribution from Liu et al. in this issue of the JCI
(16). Rather, they should stimulate future research toward the goal of better understanding the role of the kallikrein-kinin system in the various phases of lupus nephritis and other forms of autoimmunemediated glomerulonephritis.

Address correspondence to: Claudio Ponticelli, Nephrology and Dialysis Unit, IRCCS Istituto Clinico Humanitas, via Manzoni 56, 20089 Rozzano, Milano, Italy. Phone: 39-0226112952; Fax: 39-0226112951; E-mail: claudio.ponticelli@fastwebnet.it.

1. Evans, B.A., et al. 1988. Structure and chromosomal localization of the human renal kallikrein gene. Biochemistry. 27:3124-3129.

2. Riegman, P.H., et al. 1989. The prostate-specific antigen gene and the human glandular kallikrein-1 gene are tandemly located on chromosome 19. FEBS Lett. 247:123-126.

3. Bossi, F., Bulla, R., and Tedesco, F. 2008. Endothelial cells are a target of both complement and kinin system. Int. Immunopharmacol. 8:143-147.

4. Song, Q., Chao, J., and Chao, L. 1996. High level expression of human tissue kallikrein in the circulation induces hypotension in transgenic mice. Immunopharmacology. 32:105-107.

5. Margolius, H.S., Horwitz, D., Pisano, J.J., and Keiser, H.R. 1974. Urinary kallikrein excretion in hypertensive man. Relationship to sodium intake and sodium-retaining steroids. Circ. Res. 35:820-825.

6. Yu, H., et al. 2002. Association of the tissue kallikrein gene promoter with ESRD and hypertension. Kidney Int. 61:1030-1039.

7. Chao, J., Bledsoe, G., Yin, H., and Chao, L. 2006 The tissue kallikrein-kinin system protects against cardiovascular and renal diseases and ischemic stroke independently of blood pressure reduction. Biol. Chem. 387:665-675.
8. Pizard, A., et al. 2008. Genetic deficiency in tissue kallikrein activity in mouse and man: effect on arteries, heart and kidney. Biol. Chem. 389:701-706.

9. Rahman, A., and Isenberg, D.A. 2008. Systemic lupus erythematosus. N. Engl. J. Med. 358:929-939.

10. Allam, R., and Anders, H.J. 2008. The role of innate immunity in autoimmune tissue injury. Curr. Opin. Rheumatol. 20:538-544.

11. Zykova, S.N., Seredkina, N., Benjaminsen, J., and Rekvig, O.P. 2008. Reduced fragmentation of apoptotic chromatin is associated with nephritis in lupus-prone (NZB x NZW) F(1) mice. Arthritis Rheum. 58:813-25.

12. Parsa, A., et al. 2002. Association of angiotensinconverting enzyme polymorphisms with systemic lupus erythematosus and nephritis: analysis of 644 SLE families. Genes Immun. 3(Suppl. 1):S42-S46.

13. Lee, Y.H., Rho, Y.H., Choi, S.J., Ji, J.D., and Song, G.G. 2006. Angiotensin-converting enzyme insertion/ deletion polymorphism and systemic lupus erythematosus: a metaanalysis. J. Rheumatol. 33:698-702.

14. Dellalibera-Joviliano, R., Reis, M.L., and Donadi, E.A. 2001. Kinin system in lupus nephritis. Int. Immunopharmacol. 1:1889-1896.

15. Fu, Y., Du, Y., and Mohan, C. 2007. Experimental anti-GBM disease as a tool for studying spontaneous lupus nephritis. Clin. Immunol. 124:109-118.

16. Liu, K., et al. 2009. Kallikrein genes are associated with lupus and glomerular basement membranespecific antibody-induced nephritis in mice and humans. J. Clin. Invest. 119:911-923.

17. van Bavel, C.C., Fenton, K.A., Rekvig, O.P., van der Vlag, J., and Berden, J.H. 2008. Glomerular targets of nephritogenic autoantibodies in systemic lupus erythematosus. Arthritis Rheum. 58:1892-1899.

18. Weening, J.J., et al. 2004. The classification of glomerulonephritis in systemic lupus erythematosus revisited. J. Am. Soc. Nephrol. 15:241-250.

19. Bhoola, K., et al. 2001. Kallikrein and kinin receptor expression in inflammation and cancer. Biol. Chem. 382:77-89.

20. Joseph, K., and Kaplan, A.P. 2005. Formation of bradykinin: a major contributor to the innate inflammatory response. Adv. Immunol. 86:159-208. 